\title{
MARKOV-DUFFIN-SCHAEFFER INEQUALITY FOR POLYNOMIALS WITH A CIRCULAR MAJORANT
}

\author{
Q. I. RAHMAN AND G. SCHMEISSER
}

\begin{abstract}
If $p$ is a polynomial of degree at most $n$ such that $|p(x)| \leq$ $\sqrt{1-x^{2}}$ for $-1 \leq x \leq 1$, then for each $k, \max \left|p^{(k)}(x)\right|$ on $[-1,1]$ is maximized by the polynomial $\left(x^{2}-1\right) U_{n-2}(x)$ where $U_{m}$ is the $m$ th Chebyshev polynomial of the second kind. The purpose of this paper is to investigate if it is enough to assume $|p(x)| \leq \sqrt{1-x^{2}}$ at some appropriately chosen set of $n+1$ points in $[-1,1]$. The problem is inspired by a well-known extension of Markov's inequality due to Duffin and Schaeffer.
\end{abstract}

1. Introduction and statement of the results. Let us denote by $\|\cdot\|$ the maximum norm on $[-1,1]$ and by $\mathscr{P}_{n}$ the set of all polynomials of degree at most $n$. It was proved by A. A. Markov in 1889 that if $p \in \mathscr{P}_{n}$ such that $\|p\| \leq 1$, then $\left\|p^{\prime}\right\| \leq n^{2}$. Equality is attained only for $p(x) \equiv \gamma T_{n}(x)$ where $|\gamma|=1$ and

$$
T_{n}(x):=\cos (n \arccos x)=2^{n-1} \prod_{\nu=1}^{n}\left(x-\cos \left(\nu-\frac{1}{2}\right) \frac{\pi}{n}\right)
$$

is the $n$th Chebyshev polynomial of the first kind. A. A. Markov's result was extended by his brother W. A. Markov [7] as follows.

THEOREM A. If $p \in \mathscr{P}_{n}$ such that $\|p\| \leq 1$, then

$$
\left\|p^{(k)}\right\| \leq T_{n}^{(k)}(1)=\frac{n^{2}\left(n^{2}-1^{2}\right)\left(n^{2}-2^{2}\right) \cdots\left(n^{2}-(k-1)^{2}\right)}{1 \cdot 3 \cdot 5 \cdots(2 k-1)}
$$

for all $k \in \mathbf{N}$.

In 1938 Duffin and Schaeffer [13] obtained a much shorter proof. Subsequently [2] they also found the following remarkable refinement (for another proof see [3]).

TheOREM B. The conclusion (1) of Theorem A holds, if only $p \in \mathscr{P}_{n}$ and

$$
|p(\cos \nu \pi / n)| \leq 1 \text { for } \nu=0,1, \ldots, n \text {. }
$$

In addition they showed that if $E$ is any closed subset of $[-1,1]$ which does not contain all of the points $\cos (\nu \pi / n)$, then there is a polynomial $p \in \mathscr{P}_{n}$ bounded by 1 on $E$ for which (1) is not satisfied.

Theorem B has applications in the theory of Lagrange interpolation. In particular it is used to construct optimally stable formulae for numerical differentiation of functions $f$ satisfying $\|f\|<\infty$; see $[\mathbf{1}, \mathbf{6}]$.

Received by the editors August 14, 1987.

1980 Mathematics Subject Classification (1985 Revision). Primary 26D05, 30C10; Secondary 41A05, 42A05.

Key words and phrases. Markov's inequality, Chebyshev polynomial of the first kind, interpolation, circular majorant, Chebyshev polynomial of the second kind. 
In 1970 P. Turán posed the following

PROBlem. Let $p \in \mathscr{P}_{n}$ such that

$$
\|p\|_{*}:=\sup _{-1<x<1} \frac{|p(x)|}{\sqrt{1-x^{2}}} \leq 1 .
$$

How large can $\left\|p^{(k)}\right\|$ be?

For $k=1$ the answer was given in [10], for $k=2$ in [8] and for arbitrary $k \in \mathbf{N}$ in [9]. The complete result turned out to be

TheOREM C. Let $n \geq 2$ and $p \in \mathscr{P}_{n}$ such that $\|p\|_{*} \leq 1$. Then

$$
\left\|p^{(k)}\right\| \leq Q_{n}^{(k)}(1)=\frac{k}{2 k-1} \cdot \frac{2 n^{2}-4 n+k+1}{n-1} \cdot T_{n-1}^{(k-1)}(1)
$$

for all $k \in \mathbf{N}$, where

$$
Q_{n}(x):=\left(x^{2}-1\right) U_{n-2}(x)
$$

and

$$
U_{m}(x):=\frac{\sin ((m+1) \arccos x)}{\sin (\arccos x)}
$$

denotes the mth Chebyshev polynomial of the second kind. In (4) equality is attained only for $p(x)=\gamma Q_{n}(x)$ with $|\gamma|=1$.

For later reference we mention that for $k=1$ the estiamte (4) is equivalent to

$$
\left\|p^{\prime}\right\| \leq 2(n-1) \text {. }
$$

Since the time the case $k=1$ of Turán's problem was settled the question arose if in view of Theorem B of Duffin and Schaeffer it would not be sufficient to require that $|p(x)| \leq \sqrt{1-x^{2}}$ holds only at $n+1$ especially chosen points in $[-1,1]$. We investigate this question in the present paper. The answer is somewhat surprising in the sense that it is "no" for $k=1$ and "yes" for all $k>1$. More precisely, we have

THEOREM 1. Given any infinite triangular matrix

$$
\left(\begin{array}{ccccc}
x_{0}^{(0)} & & & & \\
x_{0}^{(1)} & x_{1}^{(1)} & & & \\
\vdots & \vdots & \ddots & & \\
x_{0}^{(n)} & x_{1}^{(n)} & \cdots & x_{n}^{n)} & \\
\vdots & \vdots & & \vdots & \ddots
\end{array}\right)
$$

with nodes

$$
-1 \leq x_{0}^{(n)}<x_{1}^{(n)}<\cdots<x_{n}^{(n)} \leq 1 \quad(n \in \mathbf{N})
$$

there always exists a sequence of polynomials $p \in \mathscr{P}_{n}$ and a sequence of points $\tau_{n} \in[-1,1]$ such that

$$
\left|p_{n}\left(x_{\nu}^{(n)}\right)\right| \leq\left(1-\left(x_{\nu}^{(n)}\right)^{2}\right)^{1 / 2} \quad \text { for } \nu=0,1, \ldots, n
$$

and

as $n \rightarrow \infty$.

$$
\left|p_{n}^{\prime}\left(\tau_{n}\right)\right| \geq \frac{2}{\pi}(1-o(1)) n \log n
$$

On the other hand we have the following theorem. 
THEOREM 2. Let

(9) $\xi_{0}:=-1, \quad \xi_{n}:=1 \quad$ and $\quad \xi_{\nu}:=\cos \left(\frac{2 \nu-1}{n-1} \cdot \frac{\pi}{2}\right), \quad \nu=1,2, \ldots, n-1$. If $p \in \mathscr{P}_{n}$ such that

$$
\left|p\left(\xi_{\nu}\right)\right| \leq\left(1-\xi_{\nu}^{2}\right)^{1 / 2} \quad \text { for } \nu=0,1, \ldots, n,
$$

then

$$
\left\|p^{(k)}\right\| \leq Q_{n}^{(k)}(1)
$$

for $k=2,3, \ldots$ and

$$
\left\|p^{\prime}\right\| \leq(n-1)\left(\frac{2}{\pi} \log (n-1)+3\right)=\frac{2}{\pi}(1+o(1)) n \log n
$$

as $n \rightarrow \infty$. Further, in (11) equality holds only if $p(x) \equiv \gamma Q_{n}(x)$ where $|\gamma|=1$.

REMARK 1 . If $E$ is any closed subset of $[-1,1]$ which does not contain all of the points $\xi_{0}, \xi_{1}, \ldots, \xi_{n}$, then there is a polynomial $p \in \mathscr{P}_{n}$ such that

$$
|p(x)| \leq\left(1-x^{2}\right)^{1 / 2} \text { on } E
$$

for which (11) is not satisfied.

REMARK 2. If the polynomial $p$ of Theorem 2 has real coefficients, then (11) can be extended to

$$
\left|p^{(k)}(x+i y)\right| \leq\left|Q_{n}^{(k)}(1+i y)\right|
$$

for $k=2,3, \ldots, x \in[-1,1]$ and $y \in \mathbf{R}$.

REMARK 3. Theorem 2 may be used to construct optimally stable formulae for numerical differentiation of functions $f$ satisfying $\|f\|_{*}<\infty$. Considering such a class makes sense in the case of homogeneous boundary conditions $f(-1)=f(1)=$ 0 .

Finally, we mention the following

COROLLARY. Under the hypothesis of Theorem B

$$
\left|\left(1-x^{2}\right) p^{(k+1)}(x)-2 x k p^{(k)}(x)-k(k-1) p^{(k-1)}(x)\right| \leq n Q_{n+1}^{(k)}(1)
$$

for all $k \geq 2$ and $x \in[-1,1]$.

2. Lemmas. We shall need some of the results of Duffin and Schaeffer [2] which we state as Lemmas 1, 2 and 3.

Lemma 1 [2, Lemma 1]. If

$$
q(z)=c \prod_{\nu=1}^{n}\left(z-x_{\nu}\right)
$$

is a polynomial of degree $n$ with $n$ distrinct real zeros and if $p \in \mathscr{P}_{n}$ such that

$$
\left|p^{\prime}\left(x_{\nu}\right)\right| \leq\left|q^{\prime}\left(x_{\nu}\right)\right| \quad(\nu=1,2, \ldots, n),
$$

then for $k=1,2, \ldots, n$

$$
\left|p^{(k)}(x)\right| \leq\left|q^{(k)}(x)\right|
$$

at the roots of $q^{(k-1)}(x)=0$. 
LEMma 2 [2, THEOREM I AND REMARK]. Let $q(z)$ be a polynomial of degree $n$ with $n$ distinct real zeros to the left of the point 1 and suppose that

$$
|q(x+i y)| \leq|q(1+i y)| \quad \text { for }(x, y) \in[-1,1] \times \mathbf{R} .
$$

Let $p \in \mathscr{P}_{n}$ such that

$$
\left|p^{\prime}(x)\right| \leq\left|q^{\prime}(x)\right| \text { whenever } q(x)=0 .
$$

Then for $k=1,2, \ldots, n$

$$
\left|p^{(k)}(x)\right| \leq\left|q^{(k)}(1)\right| \quad \text { for } x \in[-1,1] ;
$$

more generally

$$
\left|p^{(k)}(x+i y)\right| \leq\left|q^{(k)}(1+i y)\right| \quad \text { for }(x, y) \in[-1,1] \times \mathbf{R},
$$

if in addition $p(x)$ has real coefficients.

Lemma 3 [2, Lemma III]. The Chebyshev polynomials of the first kind satisfy

$$
\left|T_{n}(x+i y)\right| \leq\left|T_{n}(1+i y)\right| \quad \text { for }(x, y) \in[-1,1] \times \mathbf{R} .
$$

LEMMA 4. The polynomials $Q_{n}(x)$ in (5) satisfy

$$
\left|Q_{n}^{\prime}(x+i y)\right| \leq\left|Q_{n}^{\prime}(1+i y)\right| \quad \text { for }(x, y) \in[-1,1] \times \mathbf{R} .
$$

ProOF. Let $x_{1} \in(0,1]$. It follows from Lemma 3 together with an elementary geometric consideration that

$$
\left|T_{n-1}(x+i y)\right| \leq\left|T_{n-1}\left(1-x_{1}+x+i y\right)\right| \text { for } x \geq x_{1} .
$$

Hence the polynomials

$$
R(\alpha ; z):=\alpha T_{n-1}(z)+T_{n-1}\left(1-x_{1}+z\right)
$$

do not vanish in the half-plane $\left\{z \in \mathrm{C}: \operatorname{Re} z \geq x_{1}\right\}$ whenever $|\alpha|<1$. By the theorem of Gauss-Lucas the same must be true for

$$
\begin{aligned}
S(\alpha ; z):= & z^{1-(n-1)^{2}} \frac{d}{d z}\left(z^{(n-1)^{2}} R(\alpha ; z)\right) \\
= & \alpha\left((n-1)^{2} T_{n-1}(z)+z T_{n-1}^{\prime}(z)\right) \\
& +(n-1)^{2} T_{n-1}\left(1-x_{1}+z\right)+z T_{n-1}^{\prime}\left(1-x_{1}+z\right) .
\end{aligned}
$$

Setting $z=x_{1}+i y$ this implies

$$
\begin{aligned}
& \left|(n-1)^{2} T_{n-1}\left(x_{1}+i y\right)+\left(x_{1}+i y\right) T_{n-1}^{\prime}\left(x_{1}+i y\right)\right| \\
& \quad \leq\left|(n-1)^{2} T_{n-1}(1+i y)+\left(x_{1}+i y\right) T_{n-1}^{\prime}(1+i y)\right| .
\end{aligned}
$$

Obviously

$$
w:=\frac{T_{n-1}^{\prime}(1+i y)}{(n-1)^{2} T_{n-1}(1+i y)}
$$

is a point in the right half-plane. Therefore

$$
\left|1+\left(x_{1}+i y\right) w\right| \leq|1+(1+i y) w|
$$

and hence the right-hand side of (15) is majorized by

$$
\left|(n-1)^{2} T_{n-1}(1+i y)+(1+i y) T_{n-1}^{\prime}(1+i y)\right| .
$$

Since

$$
Q_{n}^{\prime}(z)=\frac{1}{n-1}\left((n-1)^{2} T_{n-1}(z)+z T_{n-1}^{\prime}(z)\right)
$$

we have verified (14) for $x \in(0,1]$. By symmetry the result extends to $x \in[-1,0)$ and by continuity to $x=0$. 
LEMMA 5. Let $p \in \mathscr{P}_{n}$ such that (10) is satisfied. Then at the roots of $Q_{n}(x)=$ 0

$$
\left|p^{\prime}(x)\right| \leq\left|Q_{n}^{\prime}(x)\right|= \begin{cases}2(n-1) & \text { if } x= \pm 1 \\ n-1 & \text { if } U_{n-2}(x)=0 .\end{cases}
$$

If equality is attained at one of the roots, then it is attained at all of them and $p(x)=\gamma Q_{n}(x)$ with $|\gamma|=1$.

PROOF. Let $R(z):=\left(z^{2}-1\right) T_{n-1}(z)$. Then

$$
R^{\prime}(z)=(n-1) Q_{n}(z)+2 z T_{n-1}(z)
$$

and hence

$$
R^{\prime}(x)= \begin{cases}2 x T_{n-1}(x) & \text { if } Q_{n}(x)=0, \\ \pm(n-1) \sqrt{1-\xi_{\nu}^{2}} & \text { if } x=\xi_{1}, \xi_{2} \ldots \xi_{n-1} .\end{cases}
$$

By Lagrange interpolation with nodes $\xi_{0} . \xi_{1} \ldots \ldots \xi_{n}$ we obtain

$$
p(x)=\sum_{\nu=1}^{n-1} \frac{p\left(\xi_{\nu}\right)}{R^{\prime}\left(\xi_{\nu}\right)} \cdot \frac{R(x)}{x-\xi_{\nu}},
$$

since $p\left(\xi_{0}\right)=p\left(\xi_{n}\right)=0$. Differentiation yields

$$
p^{\prime}(x)=\sum_{\nu=1}^{n-1} \frac{p\left(\xi_{\nu}\right)}{R^{\prime}\left(\xi_{\nu}\right)} \cdot \frac{\left(x-\xi_{\nu}\right) R^{\prime}(x)-R(x)}{\left(x-\xi_{\nu}\right)^{2}} .
$$

Using (17) we find

$$
p^{\prime}(x)=T_{n-1}(x) \sum_{\nu=1}^{n-1} \frac{p\left(\xi_{\nu}\right)}{R^{\prime}\left(\xi_{\nu}\right)} \cdot \frac{x^{2}-2 x \xi_{\nu}+1}{\left(x-\xi_{\nu}\right)^{2}} \quad \text { if } Q_{n}(x)=0 .
$$

Of course, the same representation must be true with $p(x)$ replaced by $Q_{n}(x)$. Since

$$
Q_{n}\left(\xi_{\nu}\right) / R^{\prime}\left(\xi_{\nu}\right)=\frac{1}{n-1} \quad(\nu=1,2, \ldots, n-1)
$$

we see that at the roots of $Q_{n}(x)=0$ the ratio $p^{\prime}(x) / Q_{n}^{\prime}(x)$ is a convex linear combination of the numbers $(n-1) p\left(\xi_{\nu}\right) / R^{\prime}\left(\xi_{\nu}\right)(\nu=1,2, \ldots, n-1)$ which are of modulus at most 1 by (10) and (17). This proves the inequality in (16) and the statement about occurence of equality. The values of $Q_{n}^{\prime}(x)$ at the roots of $Q_{n}(x)=0$ are easily calculated.

\section{Proofs of the results.}

PROOF OF THEOREM 1. To simplify the notation we omit the upper index of the nodes. We distinguish three cases.

Case 1. Set $\varepsilon_{n}:=1 /\left(2 n^{2} \log ^{2} n\right)$ and suppose there is more than one node in one of the intervals $\left[-1,-1+\varepsilon_{n}\right]$ and $\left[1-\varepsilon_{n}, 1\right]$.

Without loss of generality we may assume $x_{0}, x_{1} \in\left[-1,-1+\varepsilon_{n}\right]$. Then, by the Lagrange interpolation formula there exists a polynomial $p \in \mathscr{P}_{n}$ such that

$$
p\left(x_{0}\right)=\sqrt{1-x_{0}^{2}}, \quad p\left(x_{1}\right)=-\sqrt{1-x_{1}^{2}}
$$


and $p\left(x_{\nu}\right)=0(\nu=2,3, \ldots, n)$. Thus $(7)$ is satisfied, but

$$
\left|\frac{p\left(x_{1}\right)-p\left(x_{0}\right)}{x_{1}-x_{0}}\right| \geq \frac{\sqrt{1-x_{1}^{2}}}{x_{1}+1} \geq \frac{d}{d x}\left(\sqrt{1-x^{2}}\right)_{x=-1+\varepsilon_{n}}>\frac{2}{\pi} n \log n
$$

for large $n$. Hence, by the mean value theorem there exists a point $\tau \in\left[x_{0}, x_{1}\right]$ such that $p^{\prime}(\tau)>(2 n / \pi) \log n$.

Case 2. Suppose that the nodes $x_{1}, x_{2}, \ldots, x_{n-1}$ all lie in the interval $(-1+$ $\left.\varepsilon_{n}, 1-\varepsilon_{n}\right)$. Denote by

$$
\tilde{L}_{j}(x):=\prod_{\substack{\nu=1 \\ \nu \neq j}}^{n-1} \frac{x-x_{\nu}}{x_{j}-x_{\nu}} \quad(j=1,2, \ldots, n-1)
$$

the fundamental functions of Lagrange interpolation with respect to these nodes and suppose that

$$
\max _{-1 \leq x \leq 1}\left|\tilde{L}_{j}(x)\right| \geq n^{5} \quad \text { for one } j \in\{1,2, \ldots, n-1\} .
$$

In this case we find with the help of Markov's inequality that $\left|\tilde{L}_{j}(x)\right| \geq n^{5} / 2$ on a subinterval of $[-1,1]$ of length at least $1 /\left(2 n^{2}\right)$. Now already a crude estimate shows that the $j$ th Lagrange fundamental function for the complete set of nodes, namely

$$
L_{j}=\prod_{\substack{\nu=0 \\ \nu \neq j}}^{n} \frac{x-x_{\nu}}{x_{j}-x_{\nu}}
$$

attains a value of modulus bigger than $n^{3} / 8$ at a certain point $\tau \in[-1,1]$. Since $x_{j} \in\left(-1+\varepsilon_{n}, 1-\varepsilon_{n}\right)$ we have

$$
\sqrt{1-x_{j}^{2}}\left|L_{j}(\tau)\right| \geq n^{2} /(8 \log n) .
$$

Hence, for the polynomial $p(x):=\sqrt{1-x_{j}^{2}} L_{j}(x)$ which satisfies (7) we have

$$
\left|\frac{p(\tau)-p\left(x_{0}\right)}{\tau-x_{0}}\right|=\left|\frac{p(\tau)}{\tau-x_{0}}\right| \geq \frac{p(\tau) \mid}{2}>n \log n,
$$

and consequently (8) holds.

Case 3. We assume that neither Case 1 nor Case 2 holds. Then, in particular

$$
\max _{-1 \leq x \leq 1}\left|\tilde{L}_{j}(x)\right| \leq n^{5} \quad \text { for } j=1,2, \ldots, n-1 .
$$

Writing now $x_{\nu}=\cos \theta_{\nu}(\nu=0,1, \ldots, n)$ with $0 \leq \theta_{\nu} \leq \pi$ it follows from a result of Erdős [5, Theorem 2 or 3] that because of (19) the angles $\theta_{1}, \theta_{2}, \ldots, \theta_{n-1}$ are asymptotically uniformly distributed; more precisely, for $0 \leq \alpha<\beta \leq \pi$ we have

$$
\left|\sum_{\alpha \leq \theta_{\nu} \leq \beta} 1-\frac{\beta-\alpha}{\pi} n\right|<c \log ^{2} n
$$

with a constant $c>0$. Now define $L(x)=\prod_{\nu=0}^{n}\left(x-x_{\nu}\right)$ and let the node $x_{\mu}$ be chosen such that

$$
\left|L^{\prime}\left(x_{\mu}\right)\right| \geq\left|L^{\prime}\left(x_{\nu}\right)\right| \quad(\nu=0,1, \ldots, n) .
$$


If $p(x)$ is any polynomial of degree $n$, we may represent it by the Lagrange interpolation formula with respect to the nodes $x_{0}, x_{1}, \ldots, x_{n}$ and find after differentiation

$$
p^{\prime}\left(x_{\mu}\right)=\frac{p\left(x_{\mu}\right)}{2} \frac{L^{\prime \prime}\left(x_{\mu}\right)}{L^{\prime}\left(x_{\mu}\right)}+\sum_{\substack{\nu=0 \\ \nu \neq \mu}}^{n} p\left(x_{\nu}\right) \frac{L^{\prime}\left(x_{\mu}\right)}{L^{\prime}\left(x_{\nu}\right)} \cdot \frac{1}{x_{\mu}-x_{\nu}} .
$$

Since a polynomial of degree $n$ can be required to assume prescribed values at $n+1$ different points we can (in view of (21) and (22)) find $p \in \mathscr{P}_{n}$ satisfying (7) such that

$$
\left|p^{\prime}\left(x_{\mu}\right)\right| \geq \sum_{\substack{\nu=0 \\ \nu \neq \mu}}^{n} \frac{\sqrt{1-x_{\nu}^{2}}}{\left|x_{\mu}-x_{\nu}\right|}
$$

We are now going to estimate

$$
S_{1}:=\sum_{\nu=0}^{\mu-1} \frac{\sqrt{1-x_{\nu}^{2}}}{x_{\mu}-x_{\nu}} \quad \text { and } \quad S_{2}:=\sum_{\nu=\mu+1}^{n} \frac{\sqrt{1-x_{\nu}^{2}}}{x_{\nu}-x_{\mu}}
$$

from below. Setting

$$
\varphi_{j}:=\theta_{\mu}+j \frac{\pi}{n} \log ^{3} n \quad(j=0, \pm 1, \pm 2, \ldots)
$$

it follows from (20) that for $j=0, \pm 1, \pm 2, \ldots$ each of the intervals $I_{j}:=\left[\varphi_{j}, \varphi_{j+1}\right]$ which is contained in $[0, \pi]$ contains at least $(1-c / \log n) \log ^{3} n$ of the angles $\theta_{1}, \ldots, \theta_{n-1}$. Hence

$$
S_{1} \geq\left(1-\frac{c}{\log n}\right) \log ^{3} n \sum_{j>0}^{\prime} \frac{\sin \varphi_{j}}{\cos \theta_{\mu}-\cos \varphi_{j}}
$$

where the prime indicates that the summation extends only to those $j>0$ for which $I_{j} \subset[0, \pi]$. Using monotonicity we find

$$
\begin{gathered}
\frac{\pi}{n} \log ^{3} n \sum_{j>0}^{\prime} \frac{\sin \varphi_{j}}{\cos \theta_{\mu}-\cos \varphi_{j}}>\int_{\varphi_{1}}^{\pi} \frac{\sin \varphi}{\cos \theta_{\mu}-\cos \varphi} d \varphi \\
=\log \frac{1+\cos \theta_{\mu}}{\cos \theta_{\mu}-\cos \varphi_{1}}
\end{gathered}
$$

provided $\varphi_{1}<\pi$. Therefore

$$
S_{1}>\frac{n}{\pi}\left(1-\frac{c}{\log n}\right) \log \frac{1+\cos \theta_{\mu}}{\cos \theta_{\mu}-\cos \varphi_{1}}
$$

if $\varphi_{1}<\pi$ and $S_{1} \geq 0$ otherwise. Analogously we obtain

$$
S_{2}>\frac{n}{\pi}\left(1-\frac{c}{\log n}\right) \log \frac{1-\cos \theta_{\mu}}{\cos \varphi_{-1}-\cos \theta_{\mu}}
$$

if $\varphi_{-1}>0$ and $S_{2} \geq 0$ otherwise. Adding the lower bounds of $S_{1}$ and $S_{2}$ a careful analysis involving standard trigonometric formulae shows that in any case

$$
S_{1}+S_{2}>\frac{2}{\pi}(1+o(1)) n \log n
$$

for large $n$. This completes the proof of Theorem 1 . 
Proof of Theorem 2 and justification of Remark 2. Suppose (10) is satisfied and $p(x) \not \equiv \gamma Q_{n}(x)$ whenever $|\gamma|=1$. Then by Lemma 5 there exists a constant $c>1$ such that

By Lemma 1

$$
c\left|p^{\prime}(x)\right| \leq\left|Q_{n}^{\prime}(x)\right| \text { whenever } Q_{n}(x)=0 \text {. }
$$

$$
c\left|p^{\prime \prime}(x)\right| \leq\left|Q_{n}^{\prime \prime}(x)\right| \quad \text { whenever } Q_{n}^{\prime}(x)=0 .
$$

Taking Lemma 4 into account we may apply Lemma 2 with $q(z):=Q_{n}^{\prime}(z)$ and $p(x)$ replaced by $p^{\prime}(x)$. Now $(11)$ and $\left(11^{\prime}\right)$ follow immediately.

Let us now verify (12). Denoting by $\Lambda_{\nu}(x)$ the fundamental functions of Lagrange interpolation with respect to the nodes

$$
\eta_{\nu}:=-\cos \left(\frac{\nu \pi}{n-1}\right) \quad(\nu=0,1, \ldots, n-1)
$$

we have

$$
p^{\prime}(x)=\sum_{\nu=0}^{n-1} p^{\prime}\left(\eta_{\nu}\right) \Lambda_{\nu}(x)
$$

Since $\eta_{0}=-1, \eta_{n-1}=1$ whereas all other points $\eta_{\nu}$ are zeros of $U_{n-2}(x)$ we may apply Lemma 5 and obtain

$$
\left|p^{\prime}(x)\right| \leq(n-1)\left(M_{1}+2 M_{2}\right)
$$

where

$$
M_{1}:=\max _{-1 \leq x \leq 1} \sum_{\nu=0}^{n-1}\left|\Lambda_{\nu}(x)\right|
$$

and

$$
M_{2}:=\max _{-1 \leq x \leq 1}\left(\left|\Lambda_{0}(x)\right|+\left|\Lambda_{n-1}(x)\right|\right) .
$$

It is shown that in [4] that

$$
M_{1} \leq \frac{1}{n-1} \sum_{\nu=1}^{n-1} \cot \left(\frac{2 \nu-1}{n-1} \cdot \frac{\pi}{4}\right) .
$$

An explicit bound for the right-hand side is deduced in [11, pp. 17-20] which yields

$$
M_{1} \leq \frac{2}{\pi} \log (n-1)+1
$$

Finally, we calculate

$$
M_{2}=\max _{-1 \leq x \leq 1}\left|\frac{U_{n-2}(x)}{2 U_{n-2}(1)}\right|(|x-1|+|x+1|)=1 .
$$

Now (12) follows from (23)-(25).

Justification of Remark 1 . Suppose $\xi_{j} \notin E$. If $j=n$, then for sufficiently small $\varepsilon>0$

$$
p(x):=\sqrt{\frac{2}{2+\varepsilon}} Q_{n}\left(\frac{\varepsilon+(2+\varepsilon) x}{2}\right)
$$

satisfies (13), but $\left|p^{(k)}(1)\right|>Q_{n}^{(k)}(1)$. Of course, we can argue similarly if $j=0$. Suppose now $0<j<n$. Then for a sufficiently small $\delta>0$

$$
E \subset\left[-1, \xi_{j}-\delta\right] \cup\left[\xi_{j}+\delta, 1\right]=: E^{\prime}
$$


Defining

$$
R(x):=\frac{\left(x^{2}-1\right) T_{n-1}(x)}{x-\xi_{j}}, \quad S(\varepsilon, x):=Q_{n}(x)+\varepsilon R(x)
$$

and

$$
f(\varepsilon, x):= \begin{cases}S(\varepsilon, x) / \sqrt{1-x^{2}} & \text { if } x \in(-1,1) \\ 0 & \text { if } x= \pm 1\end{cases}
$$

we see that $f(0, x)$ attains its maximum modulus on $E^{\prime}$ at the points $\xi_{1}, \ldots, \xi_{j-1}$, $\xi_{j+1}, \ldots, \xi_{n-1}$. Hence for small $\varepsilon>0$ the function $f(\varepsilon, x)$ attains its maximum modulus on $E^{\prime}$ at a point $\xi(\varepsilon)$ which is close to one of these points, say $\xi_{l}$ where $l \notin\{0, j, n\}$. Calculating the root of $f^{\prime}(\varepsilon, x)=0$ which lies closest to $\xi_{l}$ we find

$$
\xi(\varepsilon)=\xi_{l}+O(\varepsilon)
$$

and

$$
|f(\varepsilon, \xi(\varepsilon))|=1+O\left(\varepsilon^{2}\right) \text { as } \varepsilon \rightarrow 0 .
$$

Hence

$$
p(x):=S(\varepsilon, x) /|f(\varepsilon, \xi(\varepsilon))|
$$

satisfies (13) but

$$
\begin{aligned}
\left|p^{(k)}(1)\right| & =\left(Q_{n}^{(k)}(1)+\varepsilon R^{(k)}(1)\right) /|f(\varepsilon, \xi(\varepsilon))| \\
& =Q_{n}^{(k)}(1)+\varepsilon R^{(k)}(1)+O\left(\varepsilon^{2}\right) \text { as } \varepsilon \rightarrow 0 .
\end{aligned}
$$

This shows that (11) is not true for small positive $\varepsilon$.

Justification of Remark 3. Let $C_{*}^{j}[-1,1]$ be the space of all $j$ times continuously differentiable functions on $[-1,1]$ such that $f( \pm 1)=0$ endowed with the norm $\|\cdot\|_{*}$. Denote by

$$
L_{n}[f]:=L\left(x_{0}, \ldots, x_{n}\right)[f]
$$

the Lagrange interpolation polynomial of $f$ with respect to the nodes $x_{0}, \ldots, x_{n}$. In "numerical differentiation"

$$
L_{n}^{(k)}[f](x):=\frac{d^{k}}{d x^{k}} L_{n}[f](x) \quad(1 \leq k \leq j)
$$

is used as an appropriate value of $f^{(k)}(x)$. The "global stability" of the operator $L_{n}^{(k)}: f \mapsto L_{n}^{(k)}[f]$ for the space $C_{*}^{j}[-1,1]$ is determined by (cf. [12])

$$
\left\|L_{n}^{(k)}\right\|_{*}:=\sup \left\{\left\|L_{n}^{(k)}[f]\right\| /\|f\|_{*}: f \neq 0, f \in C_{*}^{j}[-1,1]\right\} .
$$

In fact, if $f, \tilde{f} \in C_{*}^{j}[-1,1]$, then

$$
\left|L_{n}^{(k)}[f](x)-L_{n}^{(k)}[\tilde{f}](x)\right| \leq\left\|L_{n}^{(k)}\right\|_{*} \cdot\|f-\tilde{f}\|_{*}
$$

for $x \in[-1,1]$. Hence by Theorem 2 we have optimal stability (i.e. $\left\|L_{n}^{(k)}\right\|_{*}$ becomes smallest) for the nodes (9) and

$$
\left\|L_{n}^{(k)}\right\|_{*}=Q_{n}^{(k)}(1)=O\left(n^{2 k-1}\right) \quad \text { for } k>1
$$

whereas

$$
\left\|L_{n}^{\prime}\right\|_{*}=\frac{2}{\pi}(1+o(1)) n \log n \quad \text { as } n \rightarrow \infty
$$


With respect to the norm $\|\cdot\|$ which ignores zeros at \pm 1 we have to employ Theorem $\mathrm{B}$ and get bigger values for the corresponding quantities. Notice also that zeros at \pm 1 can be created by replacing $f$ by

$$
f_{*}(x):=f(x)-f(-1)-\frac{x+1}{2}(f(1)-f(-1)) .
$$

PROOF OF COROLlaRY. If $p$ satisfies the hypothesis of Theorem $\mathrm{B}$, then by Lemma IV in [2] the polynomial

$$
q(x):=\frac{\left(x^{2}-1\right) p^{\prime}(x)}{n-1}
$$

satisfies the hypothesis of Theorem 2. Now applying (11) with $p$ replaced by $q$ we obtain the desired inequality.

\section{REFERENCES}

1. D. L. Berman, Solution of an extremal problem of the theory of interpolation, Dokl. Akad. Nauk SSSR 87 (1952), 167-170. (Russian)

2. R. J. Duffin and A. C. Schaeffer, A refinement of an inequality of the brothers Markoff, Trans. Amer. Math. Soc. 50 (1941), 517-528.

3. R. J. Duffin and L. A. Karlovitz, The Markoff-Duffin-Schaeffer inequalities abstracted, Proc. Nat. Acad. Sci. U.S.A. 82 (1985), 955-957.

4. H. Ehlich and K. Zeller, Auswertung der Normen von Interpolntions-operatoren, Math. Ann. 164 (1966), 105-112.

5. P. Erdos, On the uniform distribution of the roots of certain polynomials, Ann. of Math. $\mathbf{4 3}$ (1942), 59-64.

6. R. Haverkamp, Zur Konvergenz der Ableitung von Interpolationspolynomen, Computing 32 (1984), 343-355.

7. W. A. Markoff, Über Polynome, die in einem gegebenen Intervalle möglichst wenig von Null abweichen, Math. Ann. 77 (1916), 218-258.

8. R. Pierre and Q. I. Rahman, On a problem of Turán about polynomials, Proc. Amer. Math. Soc. 56 (1976), 231-238.

9. ___ On a problem of Túran about polynomials II, Canad. J. Math. 33 (1981), 701-733.

10. Q. I. Rahman, On a problem of Turán about polynomials with curved majorants, Trans. Amer. Math. Soc. 163 (1972), 447-455.

11. T. J. Rivlin, The Chebyshev polynomials, Wiley, New York, 1974.

12. __ Optimally stable Lagrange numerical differentiation, SIAM J. Numer. Anal. 12 (1975), 712-725.

13. A. C. Schaeffer and R. J. Duffin, On some inequalitites of S. Bernstein and W. Markoff for derivatives of polynomials, Bull. Amer. Math. Soc. 44 (1938), 289-297.

DÉPARTEMENT de Mathématiques et de Statistique, UniVERsité de Montréal, Montréal, P. Q., H3C 3J7, CANADA

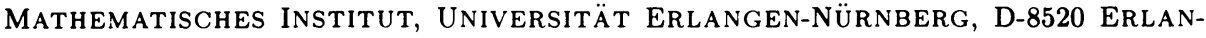
GEN, FEDERAL REPUBLIC OF GERMANY 